\title{
A WEIBULL LIMIT FOR THE RELIABILITY OF A CONSECUTIVE $\boldsymbol{k}$ - WITHIN-m-OUT-OF-n SYSTEM
}

\author{
STAVROS G. PAPASTAVRIDIS, ${ }^{*}$ University of Patras
}

\begin{abstract}
A consecutive $k$-within- $m$-out-of- $n$ system consists of $n$ identical and stochastically independent components arranged on a line. The system will fail if and only if within $m$ consecutive components, there are at least $k$ failures. Let $T_{n}$ be the system's lifetime. Then, under quite general conditions we prove that there is a positive constant $a$ such that the random variable $n^{1 / k a} T_{n}$ converges to a Weibull distribution as $n \rightarrow \infty$.
\end{abstract}

WEIBULL DISTRIBUTION

\section{Introduction}

Consecutive $k$-within- $m$-out-of- $n$ systems were introduced by Griffith (1986), as a generalization of consecutive $k$-out-of- $n$ systems (see for example Tong (1985)). Essentially, in a different context, the mathematical equivalent of a consecutive $k$-within- $m$-out-of- $n$ system, was studied much earlier (see for example, Saperstein (1973), (1975)).

A consecutive $k$-within- $m$-out-of- $n$ system consists of $n$ linearly ordered components. The $n$ components are identical and their failure times are stochastically independent. The system will fail if and only if there are $m$ consecutive components which include among them, at least $k$ failed components. Let $T$ be the time of failure of an individual component and $T_{n}$ be the time of failure of the system. Let $q$ be the failure distribution of an individual component, i.e., $q(t)=\operatorname{Pr}\{T \geqq t\}$, for $t \geqq 0$. We are interested in studying the failure distribution of the system, $\operatorname{Pr}\left\{T_{n} \leqq t\right\}$.

As Saperstein (1973), (1975) and Griffith (1986) explain, it is very difficult to compute the failure distribution of the system.

Our main result is the following limit theorem.

Theorem. Let $m \geqq k \geqq 2$, and

$$
q(t)=\lambda^{a} t^{a}+o\left(t^{a}\right)
$$

where $a, \lambda$ are positive real constants. Then

$$
\left.\operatorname{Pr}\left\{n^{(1 / k a}\right) T_{n} \leqq t\right\} \rightarrow 1-\exp \left(-(\lambda t)^{a k} \sum_{j=k}^{m}\left(\begin{array}{l}
j-2 \\
k-2
\end{array}\right)\right) \text { as } n \rightarrow \infty .
$$

For the special case of a consecutive $k$-out-of- $n$ system, this theorem has been proved by Papastavridis (1987); the proof was based on the generating function of the failure distribution, which was quite manageable for this special case.

Remark. The assumption that we made on the distribution $q$ is a very mild one. Really, since $q(0)=0$, assuming the existence of a Taylor expansion of $q$, around zero, in a right neighborhood of zero, implies the assumption of the theorem.

Received 4 January 1988; revision received 2 June 1988.

* Postal address: Department of Mathematics, University of Patras, 26110 Patras, Greece.

This paper was written while the author was visiting Bell Laboratories (MH), in the fall of 1987-88. 


\section{Proof of theorem}

The proof of our theorem is based on Theorem 2, p. 399 of Barbour and Eagleson (1984). We consider the set

$$
A=\{(i-j+1, i-j+2, \cdots, i-1, i): 1 \leqq i-j+1, i \leqq n, k \leqq j \leqq m\}
$$

which consists of $j$-tuples of numbers $1,2, \cdots, n$ which represent the component of the system in their ordering. Following the notation of Barbour and Eagleson (1984) p. 399, let $J=(i-j+1, \cdots, i) \in A$ and let $X_{J}$ be the random variable which has the value 1 if and only if components $(i-j+1)$ and $i$ are failed and there are $k$ failures among components, $i-j+1, \cdots, i$. In all other cases, $X_{J}$ has the value 0 . Let the random variables $X=\sum_{J \in A} X_{J}$. Clearly, the system fails if and only if $X$ is greater than 0 . Let $t_{n}=\operatorname{tn}^{-(1 / k a)}$, where $t \geqq 0$. We consider the system in the time interval from 0 to $t_{n}$. Clearly

$$
p_{J}=E X_{J}=q\left(t_{n}\right)^{k}\left(1-q\left(t_{n}\right)\right)^{j-k}\left(\begin{array}{l}
j-2 \\
k-2
\end{array}\right)
$$

and

$$
E X=(n-m+1) q\left(t_{n}\right)^{k} \sum_{j=k}^{m}\left(1-q\left(t_{n}\right)\right)^{j-k}\left(\begin{array}{l}
j-2 \\
k-2
\end{array}\right)+\sum p_{J}
$$

where in the second summation $J$ ranges over $j$-tuples of the numbers $1,2, \cdots, m$, only.

It is easy to see that

$$
q\left(t_{n}\right)^{k}=(\lambda t)^{a k} / n+o(1 / n)
$$

so, as $n \rightarrow \infty$ we have from (1)

$$
E X \rightarrow(\lambda t)^{a k} \sum_{j=k}^{m}\left(\begin{array}{l}
j-2 \\
k-2
\end{array}\right)
$$

because the summand $\sum p_{J}$ clearly goes to 0. By Theorem 2, p. 399 of Barbour and Eagleson (1984), the difference

$$
\operatorname{Pr}\left\{T_{n} \leqq t_{n}\right\}-(1-\exp (-E X))
$$

is bounded absolutely by

$$
\min (1,1 / E X)\left(\sum p_{J}^{2}+\sum\left(p_{J} p_{K}+E X_{J} X_{K}\right)\right)
$$

where the first summation ranges over $J \in A$ and the second one ranges over $J, K \in A$ with $J \neq K$ and $J$ and $K$ have at least one common component. Clearly we have

$$
p_{J} p_{K}=(\lambda t)^{2 a k} / n^{2}+o\left(1 / n^{2}\right), \text { for } J, K \in A
$$

and for $J, K \in A, J \neq K$ and having at least one common component, we have

$$
E X_{J} X_{K}=O\left(n^{-1-(1 / k)}\right) \text {. }
$$

That means that quantity (5) goes to 0 as $n \rightarrow \infty$. So (3) and (4) prove our theorem.

\section{Acknowledgements}

The author is grateful to Bell Laboratories (Murray Hill), where this paper was written, for the hospitality provided and for a very stimulating atmosphere. The author is also grateful to F. K. Hwang for making available to him bibliographical information about the topic of this paper. 


\section{References}

Barbour, A. D. and Eagleson, G. K. (1984) Poisson convergence for dissociated statistics. $J$. $R$. Statist. Soc. B 46, 397-402.

GRIFFITH, W. S. (1986) On consecutive $k$-out-of- $n$ failure systems and their generalizations. Reliability and Quality Control, 157-165.

PAPASTAVRIDIS, S. G. (1987) A limit theorem for the reliability of a consecutive- $k$-out-of- $n$ system. Adv. Appl. Prob. 19, 746-748.

SAPERSTEIN, B. (1973) On the occurrence of $n$ successes within $N$ Bernoulli trials. Technometrics 15, 809-818.

SaPERStein, B. (1975) Note on a clustering problem. J. Appl. Prob. 12, 629-632.

TONG, Y. L. (1985) A rearrangement inequality for the longest run with an application to network reliability. J. Appl. Prob. 22, 386-393. 